\title{
REMARKS ON METHODS FOR THE COMPUTATION OF BOUNDARY-ELEMENT INTEGRALS BY CO-ORDINATE TRANSFORMATION
}

J. SANZ-SERNA

M. DOBLARE

E. ALARCON

\section{INTRODUCTION}

It is well known that the evaluation of the influence matrices in the boundary-element method requires the computation of singular integrals.

Quadrature formulae exist which are especially tailored to the specific nature of the singularity, i.e. $\left.\log \left(x-x_{0}\right), 1 / x-x_{0}\right)$, etc. Clearly the nodes and weights of these formulae vary with the location $x_{0}$ of the singular point.

A drawback of this approach is that a given problem usually includes different types of singularities, and therefore a general-purpose code would have to include many alternative formulae to cater for all possible cases. Recently, several authors ${ }^{1-3}$ have suggested a typeindependent alternative technique based on the combination of standard Gaussian rules with non-linear co-ordinate transformations. The transformation approach is particularly appealing in connection with the p.adaptive version, where the location of the collocation points varies at each step of the refinement process.

The purpose of this paper is to analyse the technique in Reference 3. We show that this technique is asymptotically correct as the number of Gauss points increases. However, the method possesses a 'hidden' source of error that is analysed and can easily be removed.

\section{BICUBIC TRANSFORMATION}

Let

$$
I=\int_{-1}^{1} f(\eta) \mathrm{d} \eta
$$

be the integral to be evaluated, where $f$ is singular at the known point $\bar{\eta},-1<\bar{\eta}<1$. According to the method suggested in Reference 3, (1) may be rewritten as

$$
I=\int_{-1}^{\bar{\eta}} f\left(\eta_{1}\right) \mathrm{d} \eta_{1}+\int_{\bar{\eta}}^{1} f\left(\eta_{2}\right) \mathrm{d} \eta_{2}
$$


and the co-ordinates $\eta_{1}$ and $\eta_{2}$ are replaced by new variables $\xi_{1}$ and $\xi_{2},-1<\xi_{1}, \xi_{2}<1$, with

$$
\begin{aligned}
& \eta_{1}=\bar{\eta}+A\left(\xi_{1}-1\right)^{2}+B\left(\xi_{1}-1\right)^{3} \\
& \eta_{2}=\bar{\eta}+C\left(\xi_{2}+1\right)^{2}+D\left(\xi_{2}+1\right)^{3}
\end{aligned}
$$

After replacing $\eta_{1}$ and $\eta_{2}$ by $\xi_{1}$ and $\xi_{2}$, respectively, in (2) the resulting integrals are computed by the standard Gaussian rule, with $n$ nodes ( $n$ should be the same for the $\xi_{1}$ and the $\xi_{2}$ integrals).

In (3) the coefficients $A, B, C$ and $D$ are determined by the following conditions:

(i) The value $\xi_{1}=-1$ is mapped into $\eta_{1}=-1$.

(ii) The value $\xi_{2}=1$ is mapped into $\eta_{2}=1$.

(iii) If $\mu_{n}$ is the $n$th largest abscissa of the Gauss formula being employed, then $\mu_{n}$ is mapped into $\eta_{1}=\bar{\eta}-\varepsilon$, where $\varepsilon$ is a small positive number, chosen by the user.

(iv) $\xi_{2}=-\mu_{n}$ is mapped into $\eta_{2}=\bar{\eta}+\varepsilon$.

Note that the form of the expressions in (3) implies that $\eta_{1}=1, \eta_{2}=-1$ are mapped into $\xi_{1}=\bar{\eta}$, $\xi_{2}=\bar{\eta}$, respectively, and that the Jacobian of the transformation $\eta_{1}\left(\xi_{1}\right): \eta_{2}\left(\xi_{2}\right)$ vanishes at the singularity. The latter feature results in a smoothing of some types of singularities. ${ }^{2}$

The conditions (iii) and (iv) are imposed to mimic the symmetry implied in the definition of Cauchy principal-value integrals.

Owing to the combination of local smoothing and symmetry, this method can be applied to integrate many types of singularity, thus fulfilling the versatility requirement mentioned in the introduction, as shown by the numerical examples reported in Reference 3.

\section{ANALYSIS}

We study the case where the integrand $f(\eta)$ is of the form $g(\eta) /(\eta-\bar{\eta})$, with $g$ regular. Clearly it is sufficient to consider the case where $g=1 .{ }^{3}$ When $f(\eta)=1 /(\eta-\bar{\eta})$, the change of variables (3) in (2) leads formally to

$$
I^{*}=\int_{-1}^{1} \frac{2 A\left(\xi_{1}-1\right)+3 B\left(\xi_{1}-1\right)^{2}}{A\left(\xi_{1}-1\right)^{2}+B\left(\xi_{1}-1\right)^{3}} \mathrm{~d} \xi_{1}+\int_{-1}^{1} \frac{2 C\left(\xi_{2}+1\right)+3 D\left(\xi_{2}+1\right)^{2}}{C\left(\xi_{2}+1\right)^{2}+D\left(\xi_{2}+1\right)^{3}} \mathrm{~d} \xi_{2}
$$

or

$$
I^{*}=\int_{-1}^{1} \frac{2 \mathrm{~d} \xi_{1}}{\xi_{1}-1}+\int_{-1}^{1} \frac{2 \mathrm{~d} \xi_{2}}{\xi_{2}+1}+\int_{-1}^{1} R_{1}\left(\xi_{1}\right) \mathrm{d} \xi_{1}+\int_{-1}^{1} R_{2}\left(\xi_{2}\right) \mathrm{d} \xi_{2}
$$

where $R_{1}$ and $R_{2}$ are regular functions. When $I^{*}$ is computed numerically by the procedure outlined above, the approximations corresponding to the first two formal integrals in $S$ cancel by symmetry. Therefore the numerical result is given by

$$
I_{n}^{*}=Q_{n}\left[\int_{-1}^{1} R_{1}\left(\xi_{1}\right) \mathrm{d} \xi_{1}\right]+Q_{n}\left[\int_{-1}^{1} R_{2}\left(\xi_{2}\right) \mathrm{d} \xi_{2}\right]
$$

where $Q_{n}$ denotes application of the Gaussian quadrature.

On the other hand, by definition of the Cauchy principal value:

$$
\begin{aligned}
I & =\lim _{\delta \rightarrow 0} \int_{-1}^{\dot{\eta}-\delta} \frac{\mathrm{d} \eta_{1}}{\eta_{1}-\bar{\eta}}+\int_{\dot{\eta}+\delta}^{1} \frac{\mathrm{d} \eta_{2}}{\eta_{2}-\bar{\eta}} \\
& =\lim _{\sigma \rightarrow 0}\left[\int_{-1}^{\xi_{1}\left(\eta_{1}=\bar{\eta}-\delta\right)} \frac{2 A\left(\xi_{1}-1\right)+3 B\left(\xi_{1}-1\right)^{2}}{A\left(\xi_{1}-1\right)^{2}+B\left(\xi_{1}-1\right)^{3}} \mathrm{~d} \xi_{1}+\int_{\xi_{2}\left(\eta_{2}=\bar{\eta}+\delta\right)}^{1} \frac{2 C\left(\xi_{2}+1\right)+3 D\left(\xi_{2}+1\right)^{2}}{C\left(\xi_{2}+1\right)^{2}+D\left(\xi_{2}+1\right)^{3}} \mathrm{~d} \xi_{2}\right]
\end{aligned}
$$




$$
I=\lim _{\delta \rightarrow 0}\left[\int_{-1}^{\xi_{1}\left(\eta_{1}=\bar{\eta}-\delta\right)} \frac{2 \mathrm{~d} \xi_{1}}{\xi_{1}-1}+\int_{\xi_{2}\left(\eta_{2}=\bar{\eta}-\delta\right)}^{1} \frac{2 \mathrm{~d} \xi_{2}}{\xi_{2}+1}+\int_{-1}^{1} R_{1}\left(\xi_{1}\right) \mathrm{d} \xi_{1}+\int_{-1}^{1} R_{2}\left(\xi_{2}\right) \mathrm{d} \xi_{2}\right.
$$

The last limit can be easily evaluated to arrive at

$$
I=\ln \left|\frac{C}{A}\right|+\int_{-1}^{1} R_{1}\left(\xi_{1}\right) \mathrm{d} \xi_{1}+\int_{-1}^{1} R_{2}\left(\xi_{2}\right) \mathrm{d} \xi_{2}
$$

Therefore there is a term $\ln |C| A \mid$ in the true value of (7) which has no numerical counterpart in (6). The presence of this term introduces a source of error in addition to the errors stemming from using $Q_{n}$. Since

$$
\begin{aligned}
& A=-\frac{8 \varepsilon+(1+\bar{\eta})\left(\mu_{n}-1\right)^{3}}{8\left(\mu_{n}-1\right)^{2}+4\left(\mu_{n}-1\right)^{3}} \\
& C=-\frac{8 \varepsilon+(1-\bar{\eta})\left(\mu_{n}-1\right)^{3}}{8\left(\mu_{n}-1\right)^{2}+4\left(\mu_{n}-1\right)^{3}}
\end{aligned}
$$

if we let $n \rightarrow \infty$ (implying $\mu_{n} \rightarrow 1$ ), then

$$
\ln |C| A|\approx \ln | \frac{\varepsilon /\left(\mu_{n}-1\right)^{2}}{\varepsilon /\left(\mu_{n}-1\right)^{2}} \mid=0
$$

so that the source of error tends to vanish. However, for $n$ moderate, the error $\ln |C| A \mid$ can be substantial.

It is obvious that the 'hidden' source of error can be removed by choosing the co-ordinate transformation to ensure that $A=C$ rather than to satisfy the interpolation condition (iii)-(iv). A new transformation that guarantees $A=C$ has been successfully implemented by the present authors. A full description of the new transformation as well as detailed numerical comparison with existing techniques will be given elsewhere. 